\title{
Cooperative tapping: Time control under different feedback conditions
}

\author{
JǏ́i MATES \\ Ludwig-Maximilians University, Munich, Germany \\ and Czechoslovak Academy of Sciences, Prague, Czechoslovakia \\ TOMÁŠ RADIL \\ Czechoslovak Academy of Sciences, Prague, Czechoslovakia \\ and \\ ERNST PÖPPEL \\ Ludwig-Maximilians University, Munich, Germany
}

\begin{abstract}
The same isochronous tone sequence was presented simultaneously to two mutually isolated subjects. In half the trials, accentuation in this sequence was accomplished by doubling the duration of the first and then of every fourth tone; in the other half, by doubling the frequency of those tones. The subjects' task was to follow the rhythm of the resulting four-tone patterns by finger tapping to tone onsets. There were four auditory feedback (FB) conditions: (1) no FB; (2) FB from the subject's own motor responses; (3) "alien" FB from the motor responses of the other pair member who, in turn, was listening to FB from his/her own tapping; (4) mutually "crossed" FB, where each pair member listened to FB from the tapping of the other. Tap onsets regularly preceded stimulus onsets. The observed order of the amount of this anticipation (from least to greatest) was: (1) own FB, (2) no FB, (3) alien FB, and (4) crossed FB. No mutual dynamic influence between simultaneously performing subjects was detected. Anticipation was more pronounced for sequences that were accentuated by frequency rather than by duration changes. The type of accent also influenced timing of intertap intervals in the rhythmic patterns. For the frequency accent, regular timing was produced, whereas for the durational accent, shortening of the second and lengthening of the fourth (the last) intertap interval were observed. The presence and source of feedback as well as the character of accentuation are therefore relevant factors in the timing of auditorally controlled rhythmic motor behavior.
\end{abstract}

There are several possible internal sources of random error in the precise timing of repetitive movements, such as fluctuations in sensory transduction, variance in the motor system, and so forth (see, e.g., Vorberg \& Hambuch, 1984; Vos \& Ellermann, 1989; Wing, Keele, \& Margolin, 1984; Wing \& Kristofferson, 1973). The same sources also might be responsible for random and systematic errors indicating the limits in precision and accuracy of the underlying processes responsible for timing in sensorimotor synchronization. Subjective timing in synchronization of simple motor responses with repetitive sequences of stimuli has been studied by many authors over a long

This experiment was performed within the framework of cooperation between the Institute of Medical Psychology, Ludwig-Maximilians University of Munich, and the Institute of Physiology, Czechoslovak Academy of Sciences, Prague. The research was supported by Grant PO 121/13 of the Deutsche Forschungsgemeinschaft. The text was revised during the period in which the first author's research work was supported by a fellowship from the Alexander von Humboldt Foundation, Germany. Correspondence should be addressed to J. Mates, Institute of Medical Psychology, Ludwig-Maximilians University, Goethestrasse 31, D-8000 Munich 2, Germany. period of time (see reviews by Fraisse, 1978, 1982). The synchronization of finger tapping with sequences of acoustical stimuli, for example, can be conceived of as a simple model of synchronization in music performance (see, e.g., Clynes \& Walker, 1982; Fraisse, 1978, 1982; Franěk, Mates, Radil, Beck, \& Pöppel, 1991a, 1991b; Franěk et al., 1989).

Many external factors may also influence the accuracy of timing when motor acts are used to follow or reproduce repetitive stimulus sequences; these factors include the modality of stimuli (Kolers \& Brewster, 1985; Najenson, Ron, \& Behroozi, 1989), the length of interstimulus intervals (Fraisse, 1978, 1982; Peters, 1989; Pöppel, Müller, \& Mates, 1990), the lateralization of stimuli (Ilmberger, Müller, Pöppel, Mates, \& Radil, 1990), or the rhythmic structure of the stimulus sequence (Franek et al., 1991b). The rhythmic structure of a stimulus sequence can be obtained by means of accentuation of some stimuli by differentiating their specific physical aspects, since accentuation is one of the factors of grouping stimuli into temporal, or rhythmic, patterns (Fraisse, 1978).

It is probable that feedback on movement execution might be a further factor influencing stimulus-driven 
repetitive movements. The influence of feedback on performance in a synchronization task has been investigated by Kolers and Brewster (1985), who used visual stimuli and visual feedback from motor responses. The variability of performance was larger than it was in tasks without additional feedback, but the mean values were uninfluenced. Kolers and Brewster assumed that different timing mechanisms operated in different sensory modalities, so it is difficult to determine from their results what would occur if both the stimuli and the feedback were in the auditory modality. Fraisse (1982) reported lower accuracy of foot-tapping in comparison with finger-tapping; because a different motor effector was used, different proprioceptive feedback was involved. The results of other experiments demonstrate that increased proprioceptive feedback improves the anticipatory timing of motor responses (Adams \& Creamer, 1962; Schmidt \& Christina, 1969). All these experimental results support the assumption about the role of feedback information.

In the present study, we examined the effect of social feedback based on cross-linking of the motor and sensory systems of two individuals (Knight, 1987; Rosenberg \& Hall, 1958; Sauter \& K. U. Smith, 1971; Smelser, 1961; K. U. Smith \& Arndt, 1969; K. U. Smith \& Kao, 1971; T. J. Smith \& K. U. Smith, 1987a, 1987b; Wagner \& Zeaman, 1956). This is achieved by creating single or double feedback loops so that the movements of 1 subject constitute the source of sensory feedback to a 2 nd subject and vice versa. Specifically, we examined the influence of the auditory feedback signal derived from the motor performance of 1 subject on another one, both of whom were working on the same task. Additionally, the effect of their mutual interaction was investigated under a crossed feedback condition. As a control condition, the individual performance of the subject was tested in isolation from any additional signal, as well as with additional auditory feedback from his/her own performance. Systematic distortions of the temporal relationships between stimulus and response sequences typical for individual performance are mostly reflected in stimulus anticipation (see, e.g., Hary \& Moore, 1985; Kolers \& Brewster, 1985; Radil, Mates, llmberger, \& Pöppel, 1990b). The aim of this experiment was therefore to analyze whether or not these systematic distortions might be compensated by means of feedback derived from another subject, whether crossed feedback would time-lock both subjects, and whether the presence or absence of an additional external feedback loop would influence the timing of individual performance.

Thus the same stimulus sequences were presented simultaneously to 2 subjects sitting in separate rooms, their common task being to follow the stimuli by finger tapping under four different conditions. Either there was no additional auditory feedback available to the subjects at all, or the subjects were provided with additional external auditory feedback derived from their own motor responses, from the responses of the other subject, who, in turn, was provided with his or her own feedback, or the feedback was crossed between the subjects. Mutual stimulus and response timing was analyzed under these conditions.

\section{METHOD}

\section{Stimuli}

Isochronous sequences of 220 tones were used. The first and every fourth tone in a stimulus sequence were accentuated either by their being of longer duration (D sequence) or by their having a higher frequency ( $F$ sequence) in comparison with the remaining tone pulses (see Figure 1). This accentuation perceptually chunked the sequence into four-tone rhythmic patterns (Fraisse, 1978), so that each sequence comprised 55 equal patterns. The duration and the frequency of each nonaccentuated tone (the second, third, and fourth tone of each pattern) were $100 \mathrm{msec}$ and $262 \mathrm{~Hz}$, respectively. The accentuated tones (the first tone of each pattern) were either twice as long $(200 \mathrm{msec})$ or double the frequency $(524 \mathrm{~Hz})$ of the remaining tones in the patterns. Identical patterns followed one after the other continuously. The temporal intervals from onset to onset of tones inside the patterns (the interstimulus intervals), as well as the interpattern intervals, were always $500 \mathrm{msec}$. It was found in previous experiments that synchronization is most regular and the variance of interresponse intervals length minimal if the tapping frequency falls into the range of interval durations between approximately 200 and $1,000 \mathrm{msec}$ (e.g., Fraisse, 1982). Given the interstimulus interval above, one stimulus sequence of 220 tones lasted $110 \mathrm{sec}$.

\section{Procedure}

An identical stimulus sequence was presented simultaneously to a pair of subjects sitting in two acoustically shielded chambers. The subjects had no direct knowledge of each other's performance. The subject's task was to synchronize the tapping of his or her index finger on a response key with the onset of the stimuli according to the rhythm of the stimulus sequence. The subjects were asked not to let the duration of stimulus tones affect the duration of their taps. At the beginning of each sequence, the subjects had to listen without responding to the first three repetitions of the four-tone pattern, so that they could become familiar with the presented rhythm. Fraisse (1982) has reported that synchronization with repetitive patterns is usually established from the third pattern on. Thus, from

D-SEQUENCE

Figure 1. Schematic representation of the two rhythmic stimulus sequences. The rhythmic structure was created by accentuation of the first and then of every fourth tone in a sequence: (1) using tone-duration difference (D sequence); (2) using tone-frequency difference ( $F$ sequence). 
Table 1

Five Experimental Conditions as Sources for Four Data Analysis Groups

\begin{tabular}{lccl}
\hline $\begin{array}{c}\text { Experimental } \\
\text { Condition }\end{array}$ & $\begin{array}{c}\text { Feedback } \\
\text { Source } \\
\text { (Subject) }\end{array}$ & $\begin{array}{c}\text { Feedback } \\
\text { Receiver } \\
\text { (Subject) }\end{array}$ & $\begin{array}{c}\text { Data Analysis } \\
\text { Group }\end{array}$ \\
\hline No feedback & none & 1 & no feedback \\
none & 2 & no feedback \\
Own feedback & 1 & 1 & own feedback \\
Subject 1 feedback & 2 & 2 & own feedback \\
Subject 2 feedback & 1 & 1 & own feedback \\
& 1 & 2 & alien feedback \\
Crossed feedback & 2 & 1 & alien feedback \\
& 2 & 2 & own feedback \\
& 2 & 1 & crossed feedback \\
& 1 & 2 & crossed feedback \\
\hline
\end{tabular}

Note-Eighteen individual subjects performed both types of stimulus sequences (D and $F$ sequences; see Figure 1 ) in each of 5 conditions, as members of one of 9 pairs of subjects.

one stimulus sequence, we usually were able to record about 52 response patterns. A short training sequence of 20 pattern repetitions $(40 \mathrm{sec})$ preceded the experiment proper. Each member of a subject pair was instructed separately; the subjects were not informed of the experimental hypotheses.

For every tap in the sequence, the following three temporal intervals were measured and analyzed: tap duration, intertap interval (i.e., the temporal difference between successive tap onsets), and synchronization error (i.e., the temporal difference between the stimulus onset and the corresponding tap onset). If the tap onset precedes ("anticipates") the stimulus onset in time, the synchronization error is called the anticipation interval and it is represented by a negative value of the synchronization error. Positive values of the synchronization error are connected with delayed taps. We prefer this definition, in accordance with the definition used by Hary and Moore (1985) for the identical quantity named "error intervals," since it expresses the orientation in time of tap onset with respect to stimulus onset better. The "response time"' defined by Najenson et al. (1989) also indicates the same quantity, but it bears negative values in case of delayed responses.

Concurrently with the stimuli, an acoustical feedback signal was supplied to each subject. The feedback signal consisted of tones separated by pauses, with the feedback tones being triggered by tap onsets and the duration of feedback tones corresponding to tap durations. There were five different combinations of feedback signals for each pair of subjects (see Table 1). It should be noticed, however, that in all feedback conditions (i.e., even in the nofeedback condition), the tactile-kinaesthetic feedback information from the tapping responses was present. Thus, the synchronization could always be judged on the basis of this information.

The order of the experimental conditions was randomized among subject pairs. In each experimental condition, both the $\mathrm{D}$ sequence and the $F$ sequence were used, also in a random order. Therefore, 10 different trials were presented altogether. Each subject pair performed all 10 trials in one session, with the duration of a session being approximately $40 \mathrm{~min}$.

\section{Apparatus}

The stimulus tone sequence was controlled and the responses were monitored by a special program on an IBM-PC-compatible computer (Mates, 1990). The temporal resolution of interval measurement was $250 \mu \mathrm{sec}$. The stimulus sequences were generated with the internal sound generator of the computer, producing a rectangular signal of a given frequency, and they were presented to the subjects over headphones. The signal was amplified to a comfortable level for each subject individually and then kept constant throughout the experiment.

The acoustical feedback signals were produced by an external tone generator triggered directly by the response keys. The frequency of the feedback tones was always equal to $2000 \mathrm{~Hz}$, a frequency substantially higher than that of the stimulus itself. The feedback signal was supplied to each subject by means of a loudspeaker, which was positioned at a distance of approximately $1 \mathrm{~m}$ from the subject. The response keys themselves produced no sound and were identical to those commonly used in computer keyboards; this eliminated possible false response detection, which can result from mechanical switch bouncing.

\section{Subjects}

Nine paid subject pairs (i.e., 18 subjects) participated in the experiment ( 6 females, 12 males, from 26 to 55 years old). Sex and age were not taken into consideration when the pairs were formed.

\section{RESULTS}

From every individual response sequence, the first and the last pattern as well as incidental incomplete response patterns were removed before data processing. If an invalid pattern was removed from the response sequence of one member of a pair, the pattern of the same sequential order was also removed from the response sequence of the other member of the pair. The sequential correspondence of responses of both pair members was thus preserved. All response sequences of each pair recorded in the 10 trials were adjusted to the minimal common length by removing a necessary number of response patterns from the beginnings of longer sequences. Thus the statistics from all experimental conditions for both subjects of a pair were based on the same number of measurements. The numbers of response patterns used for the nine subject pairs were $48,43,45,45,46,37,45,49$, and 47 , respectively.

The data were combined for evaluation into four groups, depending on the type of auditory feedback presented (see Table 1). The four groups were: no feedback (NFB), own feedback (OFB), alien feedback (AFB), and crossed feed- 
Table 2

Degrees of Freedom, $F$ Ratios and Probability Levels of Within-Subject Effects in Repeated Measures Analyses of Variance of Intertap Intervals (ITI), Tap Durations (TD), and Synchronization Errors (SE)

\begin{tabular}{|c|c|c|c|c|c|c|c|}
\hline \multirow[b]{2}{*}{ Effect } & \multirow[b]{2}{*}{$d f$} & \multicolumn{2}{|c|}{ ITI } & \multicolumn{2}{|c|}{ TD } & \multicolumn{2}{|c|}{$\mathrm{SE}$} \\
\hline & & $F$ & $p^{*}$ & $F$ & $p$ & $F$ & $p$ \\
\hline ST & 1,17 & 0.974 & .337 & 0.330 & .573 & 5.617 & .030 \\
\hline $\mathrm{FC}$ & 3,51 & 0.821 & .471 & 0.215 & .886 & 11.730 & $<.001$ \\
\hline TP & 3,51 & 0.734 & .516 & 15.329 & $<.001$ & 0.764 & .519 \\
\hline $\mathrm{ST} \times \mathrm{FC}$ & 3,51 & 1.254 & .299 & 2.519 & .068 & 2.078 & .115 \\
\hline $\mathrm{ST} \times \mathrm{TP}$ & 3,51 & 5.640 & .007 & 9.060 & $<.001$ & 5.932 & .001 \\
\hline $\mathrm{FC} \times \mathrm{TP}$ & 9,153 & 1.075 & .380 & 0.955 & .479 & 1.054 & .400 \\
\hline $\mathrm{ST} \times \mathrm{FC} \times \mathrm{TP}$ & 9,153 & 0.896 & .464 & 0.823 & .596 & 0.934 & .497 \\
\hline
\end{tabular}

Note-ST, sequence type; FC, feedback condition; TP, tap position. *Greenhouse-Geisser's adjusted probability.

back (CFB). Because of the likely within-trial correlations between the successive intervals measured in a stream of repetitive responses, the trial means were used in the statistical analyses. Such a correlation may result from an error compensation mechanism or from the properties of repetitive responding that follow from the timing model developed by Wing and Kristofferson (1973; see also Mates, 1991; Vos \& Ellermann, 1989). Correlations between successive temporal parameters of a sequence of repetitive responses have already been observed in earlier experimental data (see, e.g., Franěk et al., 1991b; Kolers \& Brewster, 1985; Peters, 1989; Vos \& Ellermann, 1989).

\section{Intertap Intervals}

When the data were pooled for all subjects and all taps in the patterns, there was remarkably little variation in the duration of intertap intervals corresponding to interstimulus intervals (always $500 \mathrm{msec}$ ). The intraindividual standard deviations of intertap intervals varied in the range from 15 to $50 \mathrm{msec}$. Because a synchronization tapping task was used, the total mean value of intertap intervals equaled the duration of the interstimulus interval; otherwise there would have been a cumulative increase of synchronization errors.

Detailed analysis, which took into account the sequential position of taps in the patterns, was done with a repeated measures analysis of variance (ANOVA). The type of experimental sequence (D or $\mathrm{F} ; d f=1$ ), type of feedback condition (NFB, OFB, AFB, CFB; $d f=3$ ) and position of the tap (T) in the patterns $(\mathrm{T} 1-\mathrm{T} 4 ; d f=3$ ) were selected as trial (within-subject) factors, with subjects' mean intertap intervals computed over each trial as replicates $(d f=17)$. The hypothesis of homogeneity of withincell variances, on the basis of Bartlett's test, was rejected $\left[\chi^{2}(31)=77.53, p<.01\right]$, so the Greenhouse-Geisser's adjusted probabilities were used to judge the significance levels. The results of the ANOVA are presented in Table 2 . There were no significant main effects. The only significant interaction occurred between sequence type and tap position in the patterns. The total mean values of intertap intervals grouped according to the detected differences are displayed in Figure 2.
To preserve a one-to-one correspondence of measures by one subject from different levels of within-subject (trial) factors, single-degree-of-freedom contrasts, instead of any multiple comparison test (Tukey, etc.), were used for all post hoc comparisons. Post hoc analysis of withinsubject differences showed no significant effect of tap position inside the patterns for the D sequence $(p>.05)$ or for the $F$ sequence $(p>.05)$. Within-subject differences between the two sequence types were significant for the second and the fourth taps in the patterns $[F(1,17)=14.76$ for $\mathrm{T} 2$ and 13.51 for $\mathrm{T} 4, p<.01]$ and nonsignificant for the first and third taps $(p>.05)$. Further analysis showed (see Figure 2) that the second intertap interval produced in the D sequence was signifi-

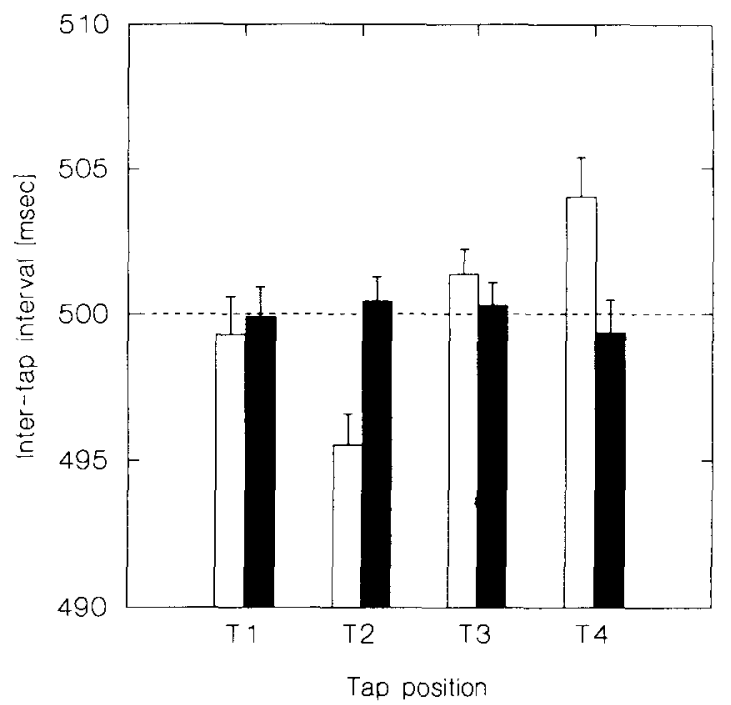

Figure 2. Total average values with between-trial-blocks standard errors of the mean of intertap intervals, plotted for both stimulus sequences (empty bars, D sequence; filled bars, F sequence) and for the four consecutive tap positions in the rhythmic patterns (T1 to T4) separately. Subjects' trial means were pooled over all feedback conditions, which revealed no significant effect on duration of intertap intervals. The dashed line represents the interstimulus interval (500 msec). 
cantly shorter than that produced in the $\mathrm{F}$ sequence, and also that it was shorter than the duration of the interstimulus interval $(500 \mathrm{msec})$. The mean duration of the second intertap interval $(495.4 \mathrm{msec})$ was significantly less than $500 \mathrm{msec}$ [one-sample $t$ test; $t(17)=2.35, p<.05$ ], being the (nonsignificantly) shortest in the patterns. This shortening was compensated mostly by lengthening of the fourth (i.e., the last) intertap interval in the patterns, whose mean for the D sequence $(504.1 \mathrm{msec})$ was significantly longer than the mean for the $\mathrm{F}$ sequence and (nonsignificantly) longer than $500 \mathrm{msec}$ [one-sample $t$ test; $t(17)=$ $1.88, p>.05]$. No significant lengthening of the first intertap interval "filled" in the D sequence with the accentuated double-lasting tone could be observed in comparison with its required duration. No significant deviations of intertap intervals from the duration of the interstimulus interval were found inside the patterns for the sequence accentuated by the higher frequency of the first tone in the patterns ( $F$ sequence).

The individual results, pooled according to the significant effects observed in the preceding analysis, are shown in Figure 3. Since there were no effects of the feedback condition and since different individual strategies of timing of intertap intervals together with the influence of type of accentuation on them have already been investigated (Franěk et al., 1991b), no other tests on interindividual differences were performed.

\section{Tap Durations}

To investigate possible factors influencing these intervals, a repeated measures ANOVA with the same factorial design as that used for the intertap intervals was performed. On the basis of a Bartlett's test, the hypothesis of overall within-cell homogeneity of variances was accepted $\left[\chi^{2}(31)=5.46, p>.05\right]$. The results of the ANOVA are presented in Table 2 . The main effect of tap position in the patterns and the interaction between sequence type and tap position were significant. The total mean values of tap durations grouped according to the detected differences are displayed in Figure 4.

Post hoc within-subject comparisons showed a gradual decrease of tap duration in the patterns. The values of the $F$ ratio and corresponding probability levels are shown in Table 3. There were no within-subject differences between the two types of sequences for any tap position in the patterns $[F(1,17), p>.05]$. Significant within-subject differences with respect to the position of tap inside the patterns were found for the $\mathrm{D}$ sequence $[F(3,51)=12.68$, $p<.01]$ as well as for the $F$ sequence $[F(3,51)=3.96$, $p<.05]$. The relationship between tap durations inside the patterns for the $D$ sequence was the same as it was for the global data. For the $F$ sequence, the duration of only the fourth tap was significantly different (shorter) than the duration of the other taps in the patterns. The values of the $F$ ratio and corresponding probability levels of the preceding two tests are also shown in Table 3.
The individual results, pooled according to the significant effects observed in the preceding analysis, are shown in Figure 5. Since there were no effects of the feedback condition, and since interindividual differences in tap durations as a consequence of different musical skill have already been studied (Franěk et al., 1991a), no other tests on interindividual differences were performed.

\section{Synchronization Errors}

The influence of the various feedback conditions on the synchronization errors (i.e., the temporal differences between stimulus onsets and corresponding tap onsets) was analyzed in a repeated measures ANOVA with the same factorial design as that for intertap intervals, in order to find out more about the variables influencing these intervals. The hypothesis of overall within-cell homogeneity of variances was retained on the basis of a Bartlett's test $\left[\chi^{2}(31)=35.47, p>.05\right]$. The results of the ANOVA are presented in Table 2 . A significant main effect was found for both sequence type and feedback condition. The interaction between sequence type and tap position was also significant. The total mean values of synchronization errors, grouped according to the detected differences, are displayed in Figure 6.

Comparison of the two types of stimulus sequences showed that anticipation intervals were longer for the accentuation by frequency of the first tone in the patterns (F sequence; $M=-69 \mathrm{msec}$ ) than for the accentuation by duration (D sequence; $M=-59 \mathrm{msec}$ ) (see Figure 6). Post hoc within-subject comparison of the influence of different feedback conditions showed that feedback conditions AFB and CFB resulted in a significant lengthening of these intervals in comparison with the conditions NFB and OFB. The values of the $F$ ratio and corresponding probability levels are shown in Table 4 . Since there was no interaction between sequence type and feedback condition, both of which revealed significant main effects, we conclude that the influence of feedback conditions on synchronization errors was similar for both sequence types. With regard to the interaction between sequence type and tap position, within-subject differences between the two types of sequences were significant for the first and second taps in the patterns $[F(1,17)=11.63$ for $\mathrm{T} 1$, $p<.01$, and 6.44 for $\mathrm{T} 2, p<.05]$ and nonsignificant for the third and fourth taps $(p>.05)$. Significant withinsubject differences with respect to the position of taps inside the patterns were found for the $\mathrm{D}$ sequence $[F(3,51)=$ $2.87, p<.05$ ], the first and the second taps being associated with a significantly shorter anticipation than was the third one. The values of the $F$ ratio and corresponding probability levels are shown in Table 5. No differences according to the tap position were found for the $F$ sequence $(p>.05)$.

The individual results, pooled according to the significant main effects observed in the preceding analysis, are shown in Figure 7. Using a Tukey's post hoc compari- 


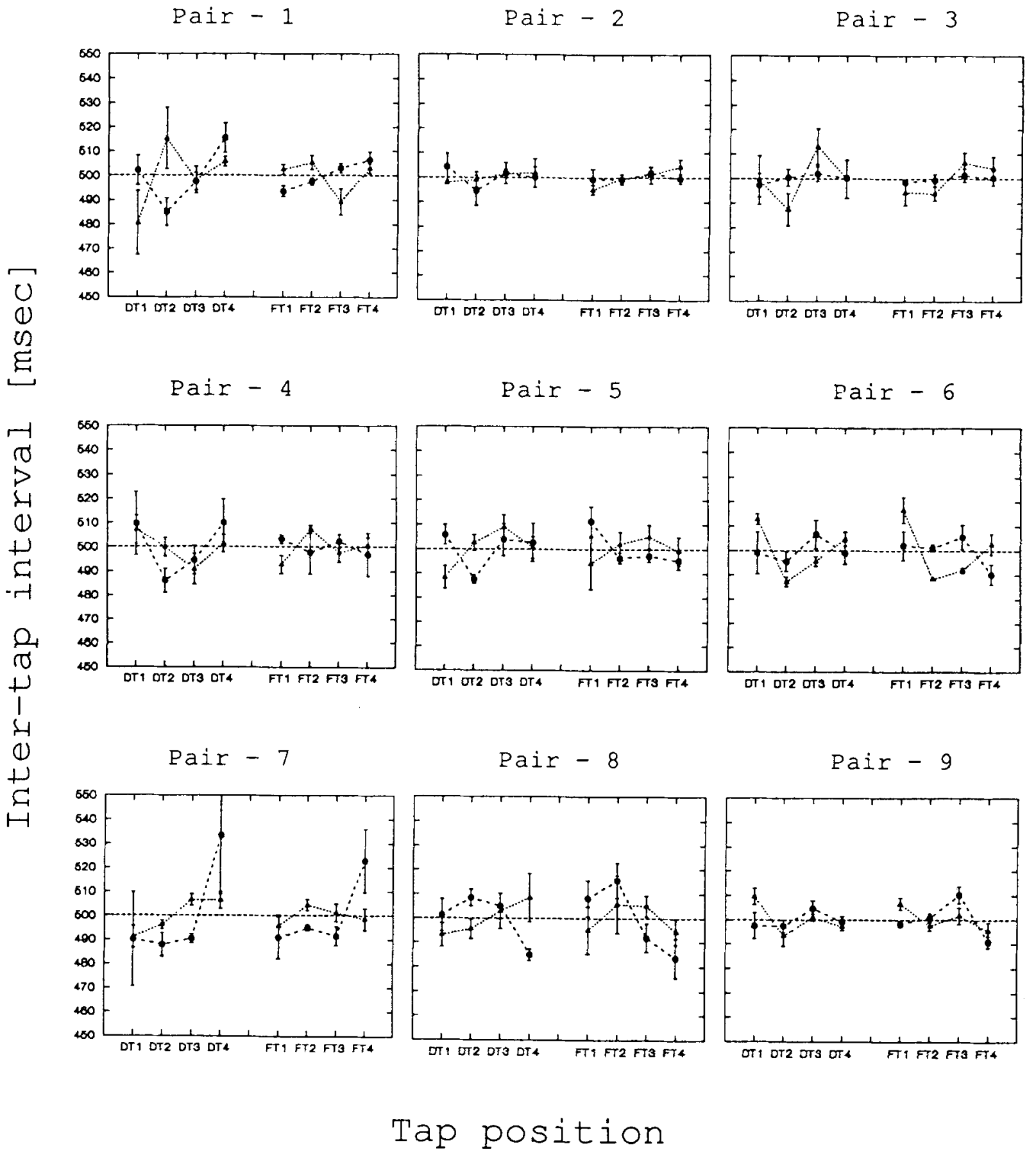

Figure 3. Individual means with between-trial-blocks standard deviations of intertap intervals plotted for both stimulus sequences and for the four consecutive tap positions in the rhythmic patterns separately (DT1-DT4, Taps 1-4 for the D sequence; FT1-FT4, Taps 1-4 for the F sequence). The data are grouped according to the experimental pairs (Pairs 1-9; circles, Subject 1; triangles, Subject 2 in one pair). Subjects' trial means were pooled over all feedback conditions, which revealed no significant effect on the duration of intertap intervals. The dashed line represents the interstimulus interval (500 msec). (Tick marks on the abscissas are not a scale; points are connected with lines to aid the eye.) 


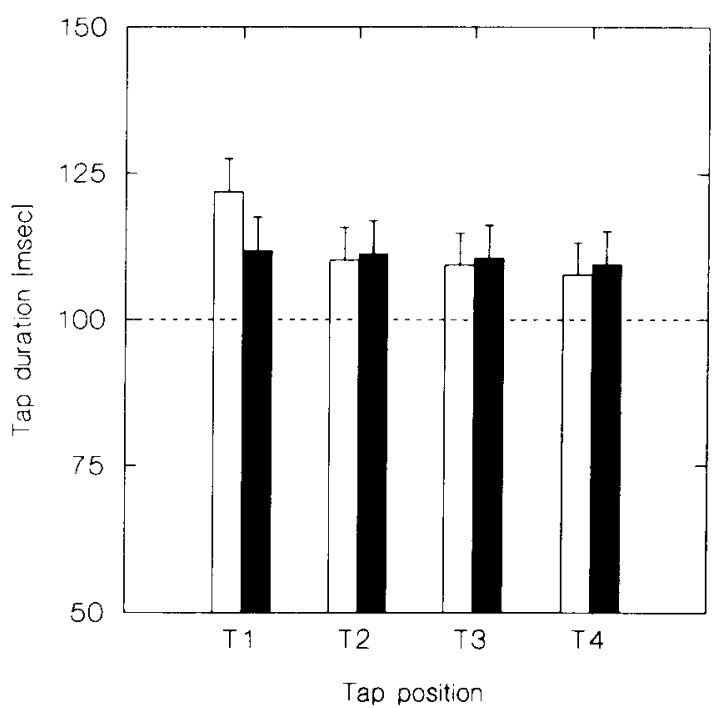

Figure 4. Total average values with between-trial-blocks standard errors of the mean of tap durations plotted for both stimulus sequences (empty bars, D sequence; filled bars, F sequence) and for the four consecutive tap positions in the rhythmic patterns (T1-T4) separately. Subjects' trial means were pooled over all feedback conditions, which revealed no significant effect on tap durations. The corresponding stimulus-tone duration was for the first tone in the D sequence equal to $200 \mathrm{msec}$; otherwise it was 100 msec (represented by the dashed line). (Cf. Figure 1.)

son, interindividual differences of synchronization errors were found $(p<.001)$. The subjects' total means and between-trials standard deviations are shown in Figure 8.

The series of successive anticipation intervals in each trial were also tested with simple linear regression to examine trends in their duration during the sequence. With the use of the $t$ statistic, the trend slopes were found to be significantly positive in $49 \%$ of cases for the D sequence and in $44 \%$ of cases for the F sequence $(p<.05)$.
The possible factors influencing the slopes of these trends were investigated by means of a repeated measures ANOVA. Type of experimental sequence (D or F; $d f=1)$ and type of feedback condition (NFB, OFB, AFB, CFB; $d f=3$ ) were selected as trial (within-subject) factors with the slopes of trends computed in each trial for all subjects as replicates $(d f=17)$. The hypothesis of homogeneity of within-cell variances, on the basis of a Bartlett's test, was rejected $\left[x^{2}(7)=15.89, p<.05\right]$, so again the Greenhouse-Geisser's adjusted probabilities were used to judge the significance levels. There was no significant main effect of sequence type $(p>.05)$ or of feedback condition $(p>.05)$, nor was their interaction significant $(p>.05)$. The mutual dependencies between slopes of trends of series of anticipation intervals for the simultaneously performing members of a subject pair were tested by using correlation coefficients computed for all pairs of subjects and all experimental conditions. These correlations were not significant for any feedback condition or for any sequence.

For conditions AFB and CFB for both the D sequence and the $F$ sequence, the values of cross-correlation functions (for lag equal to 0 and 1 ) between the series of successive anticipation intervals of pair members from each experimental pair in one trial were also computed. No systematic significant cross-correlations were found between pairs of subjects in the two feedback conditions or with the $D$ sequence and the $F$ sequence.

\section{DISCUSSION}

The present experiment, in a sense, represents a model of a maximally simplified musical duet with and without feedback between both performers, with the computer providing the external timing template and to a certain extent playing the role of a conductor. Fraisse (1982) hypothesized that the unity of musicians playing together is possible only when they are capable of anticipation. It is obvious

Table 3

F Ratios and Probability Levels of Post Hoc Within-Subject Comparison of Tap Durations According to Tap Position (1-4) for Both Sequences Together and for the D and F Sequences Separately

\begin{tabular}{|c|c|c|c|c|c|c|}
\hline \multirow{3}{*}{$\begin{array}{c}\text { Tap } \\
\text { Position }\end{array}$} & \multicolumn{6}{|c|}{ Tap Position } \\
\hline & \multicolumn{2}{|c|}{4} & \multicolumn{2}{|c|}{3} & \multicolumn{2}{|c|}{2} \\
\hline & $\boldsymbol{F}$ & $p$ & $F$ & $p$ & $F$ & $p$ \\
\hline \multicolumn{7}{|c|}{ Both Sequences } \\
\hline $\begin{array}{l}1 \\
2 \\
3\end{array}$ & $\begin{array}{l}18.330 \\
14.548 \\
12.471\end{array}$ & $\begin{array}{l}.001 \\
.001 \\
.003\end{array}$ & $\begin{array}{r}15.564 \\
2.513\end{array}$ & $\begin{array}{l}.001 \\
.131\end{array}$ & 12.648 & .002 \\
\hline \multicolumn{7}{|c|}{ D sequence } \\
\hline $\begin{array}{l}1 \\
2 \\
3\end{array}$ & $\begin{array}{r}14.037 \\
9.664 \\
8.687\end{array}$ & $\begin{array}{l}.002 \\
.006 \\
.009\end{array}$ & $\begin{array}{r}12.868 \\
1.629\end{array}$ & $\begin{array}{l}.002 \\
.219\end{array}$ & 11.521 & .003 \\
\hline \multicolumn{7}{|c|}{ F sequence } \\
\hline $\begin{array}{l}1 \\
2 \\
3\end{array}$ & $\begin{array}{l}6.070 \\
9.234 \\
7.776\end{array}$ & $\begin{array}{l}.025 \\
.007 \\
.013\end{array}$ & $\begin{array}{l}1.789 \\
1.724\end{array}$ & $\begin{array}{l}.199 \\
.207\end{array}$ & 0.571 & .460 \\
\hline
\end{tabular}

Note-The degrees of freedom in all cases are 1,17. Single-degree-of-freedom contrasts were used for pairwise comparisons. 
Pair -1

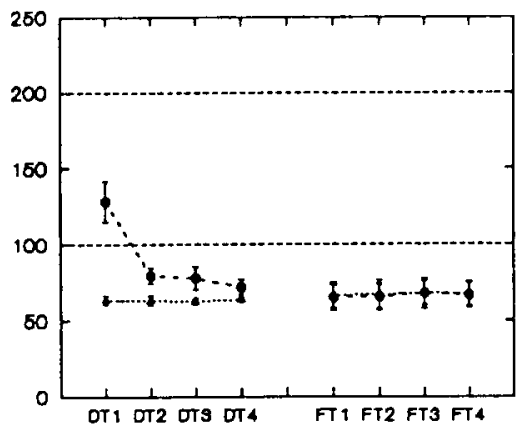

Pair - 2

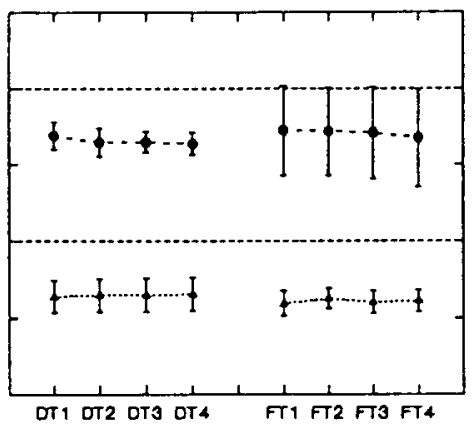

Pair - 3

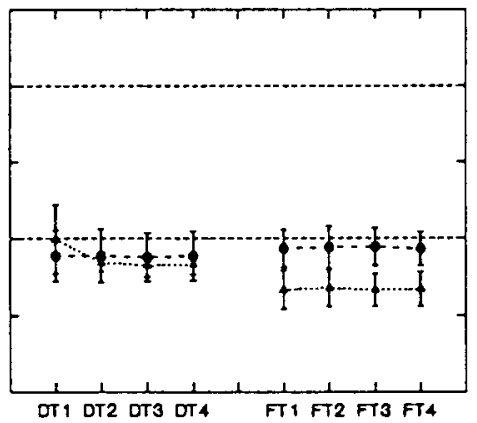

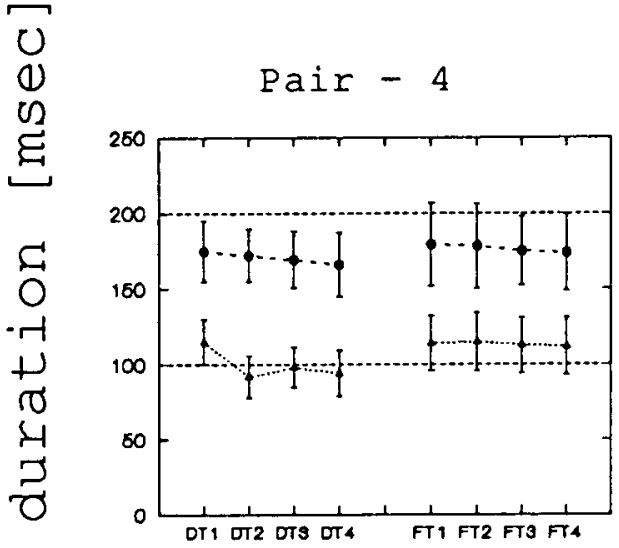
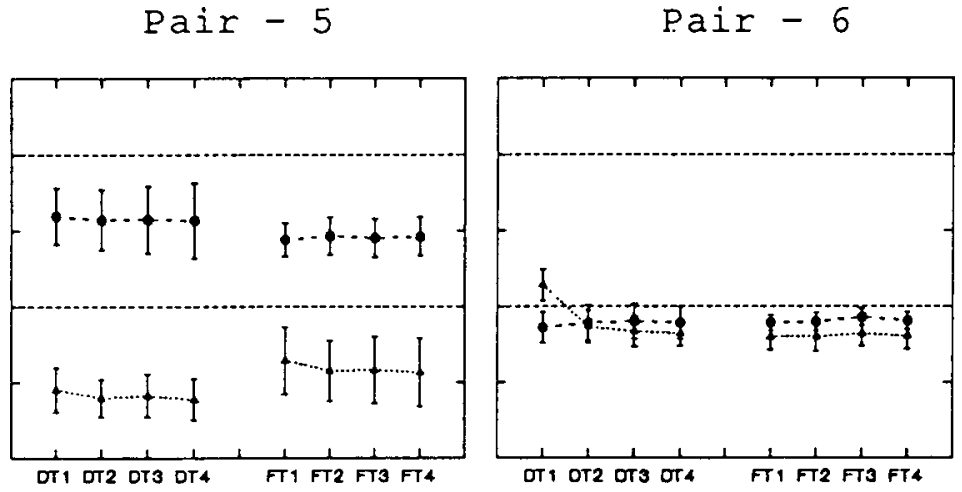

$\Omega$

$\sigma$

E-1

Pair - 7

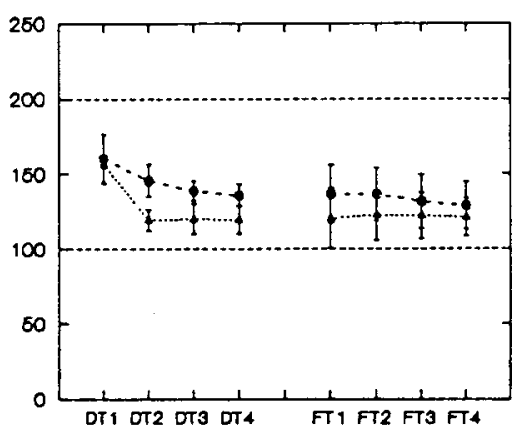

Pair - 8

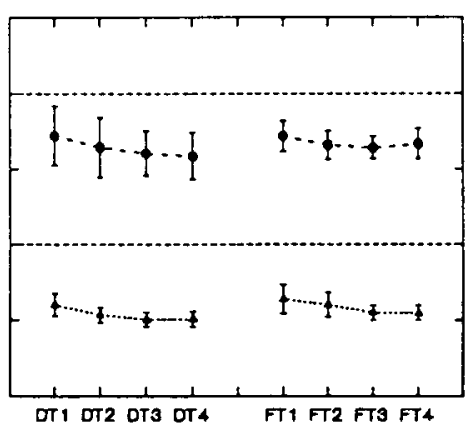

Pair - 9

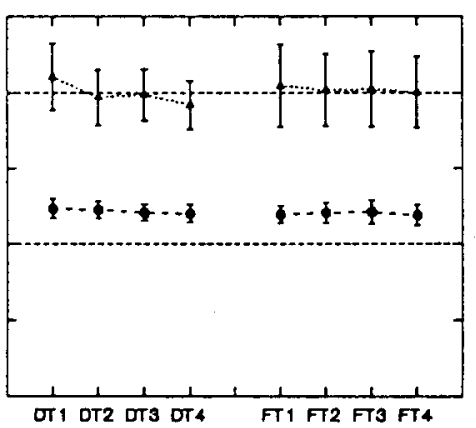

\section{Tap position}

Figure 5. Individual means with between-trial-blocks standard deviations of tap durations plotted for both stimulus sequences and for the four consecutive tap positions in the rhythmic patterns separately (DT1-DT4, Taps 1-4 for the D sequence; FT1-FT4, Taps 1-4 for the F sequence). The data are grouped according to the experimental pairs (Pairs 1-9; circles, Subject 1; triangles, Subject 2 in one pair). Subjects' trial means were pooled over all feedback conditions, which revealed no significant effect on tap durations. The corresponding stimulus-tone duration was for the first tone in the $D$ sequence equal to 200 msec; otherwise it was 100 msec (represented by the dashed lines). (Cf. Figure 1.) (Tick marks on the abscissas are not a scale; points are connected with lines to aid the eye.) 


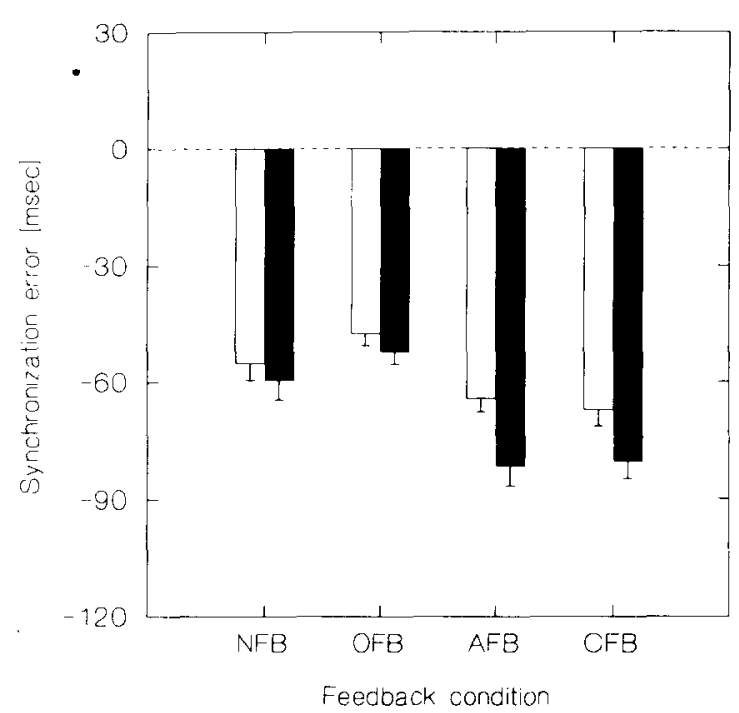

Figure 6. Total average values with between-trial-blocks standard errors of the mean of synchronization errors plotted for both stimulus sequences (empty bars, D sequence; filled bars, $F$ sequence) and for the four feedback conditions (NFB, C . ?, AFB, CFB) separately. Subjects' trial means were pooled over all consecutive tap positions in the rhythmic patterns, which revealed no significant main effect on synchronization errors. The dashed line represents the onset of stimuli; the zero level means perfect synchronization; and the area below this level corresponds to responses in advance of stimulus presentation.

Table 4

F Ratios and Probability Levels of Post Hoc Within-Subject Comparison of Synchronization Errors According to the Feedback Condition (NFB, OFB, AFB, CFB) for Both Sequences Together

\begin{tabular}{crrrrrrr}
\hline & \multicolumn{2}{c}{ CFB } & & \multicolumn{2}{c}{ AFB } & & \multicolumn{2}{c}{ OFB } \\
\cline { 2 - 5 } & \multicolumn{1}{c}{$F$} & $p$ & $F$ & $\underline{p}$ & $F$ & $p$ \\
\hline NFB & 7.790 & .013 & & 10.852 & .004 & 2.458 & .135 \\
OFB & 17.566 & .001 & 36.746 & $<.001$ & & \\
AFB & 0.048 & 829 & & & & &
\end{tabular}

Note-The degrees of freedom in all cases are $1, \overline{17}$. Single-degree-offreedom contrasts were used for pairwise comparisons. CFB, crossed feedback; NFB, no feedback; AFB, alien feedback; OFB, own feedback. that not only the capability of anticipation, but also the ability to synchronize one's own activity with the activity of other players, is the necessary condition of unity. In this study, the effect of social feedback, or "cross-yoking" (see Smith \& Kao, 1971) the sensory and motor systems of two individuals, performing identical tasks, by means of feedback loops, was examined. The results showed that unior bilateral cooperation of the type adopted in our experiment neither contributed to more accurate performance of the subjects (in the sense of reducing differences between stimulus and response timing) nor mutually synchronized the activity of both subjects. Thus, in the "social feedback" paradigm used in this study, which differs from those mentioned in the introduction mostly in the impossibility of adding up the effort of both participants in a direct way and in the lack of any type of learning and apparent adaptive behavior, no signs of real collaboration between the subjects could be found. Also, we did not observe the higher types of cooperation or competition detectable in and usually studied by means of two-person mixed-motive games (Gallo \& McClintock, 1965; Marwell \& Schmitt, 1975; Rapoport \& Chammak, 1965; Smead, 1972; Wichman, 1970).

The significance of feedback in musical performance is a complex matter that has been studied relatively little (Shaffer, 1984). The stimulus sequences in experiments like ours should be more multifarious (multitonal and with more durations) to study this problem more adequately. On the basis of our observations, we would expect that the more complex a musical piece is (complex time structure and more musicians playing together), the greater the anticipation necessary in order to obtain subjectively acceptable synchronization.

The results showed that the different feedback conditions used in our experiment primarily influenced the temporal difference between the stimulus onsets and the corresponding tap onsets (synchronization error), with the latter ones usually preceding the former ones. The other measured temporal characteristics of the subjects' performance did not vary systematically with respect to the social feedback conditions adopted.

Table 5

F Ratios and Probability Levels of Post Hoc Within-Subject Comparison of Synchronization Errors According to Tap Position (1-4) for the D Sequence

\begin{tabular}{|c|c|c|c|c|c|c|}
\hline \multirow{3}{*}{$\begin{array}{c}\text { Tap } \\
\text { Position }\end{array}$} & \multicolumn{6}{|c|}{ Tap Position } \\
\hline & \multicolumn{2}{|c|}{4} & \multicolumn{2}{|c|}{3} & \multicolumn{2}{|c|}{2} \\
\hline & $F$ & $p$ & $F$ & $p$ & $F$ & $p$ \\
\hline 1 & 2.737 & .116 & 7.674 & .013 & 0.096 & .761 \\
\hline 2 & 1.397 & .253 & 5.325 & .034 & & \\
\hline 3 & 0.972 & .338 & & & & \\
\hline
\end{tabular}

Note-The degrees of freedom in all cases are 1,17. Single-degree-of-freedom contrasts were used for pairwise comparisons. 

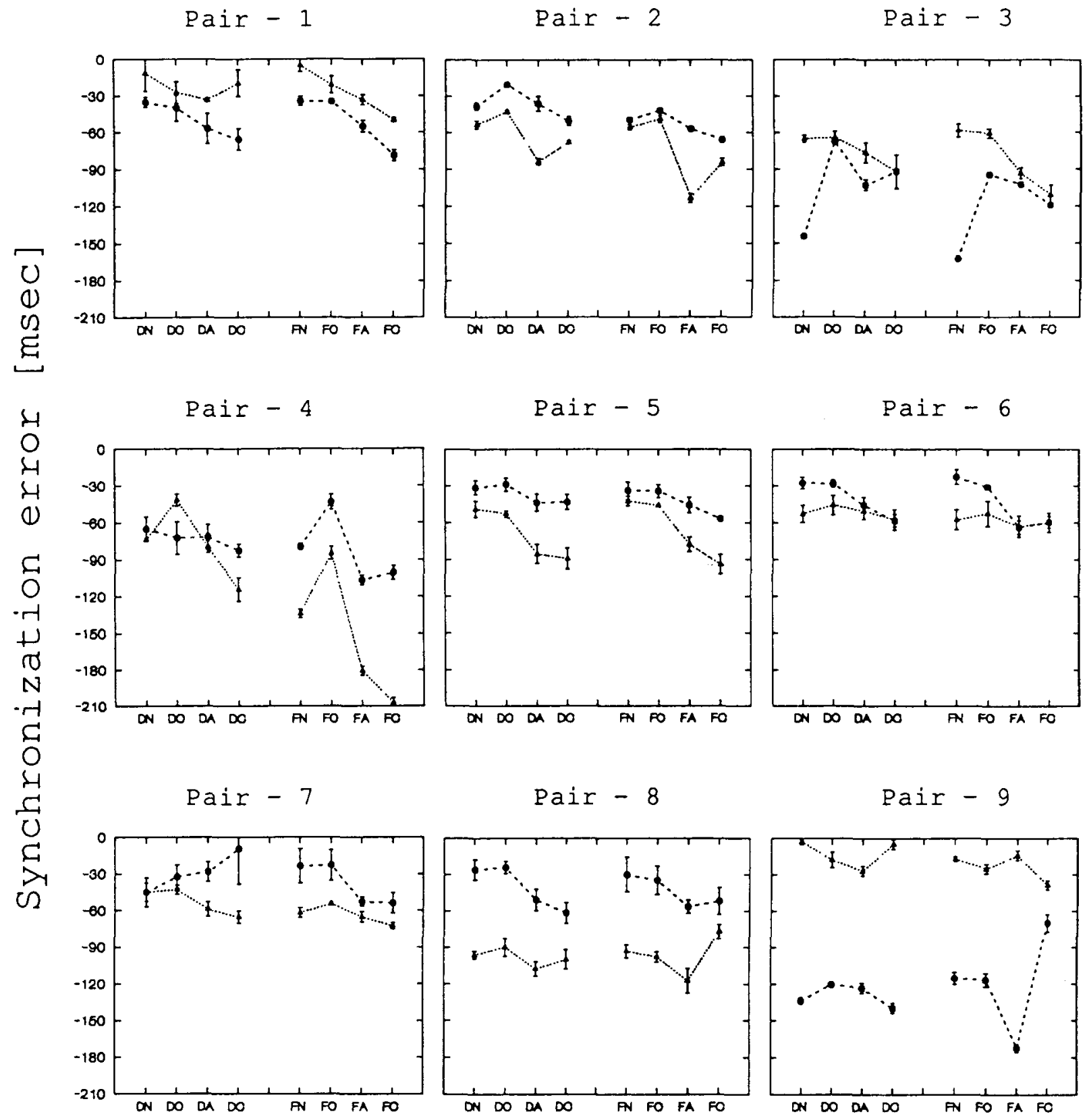

\section{Experimental condition}

Figure 7. Individual trial means with between-trial-blocks standard deviations of synchronization errors plotted for both stimulus sequences and for the four feedback conditions separately (DN, DO, DA, DC: Conditions NFB, OFB, AFB, and CFB for the D sequence; FN, FO, FA, FC: Conditions NFB, OFB, AFB, and CFB for the F sequence). The data are grouped according to the experimental pairs (Pairs 1-9; circles, Subject 1; triangles, Subject 2 in one pair). Subjects' trial means were pooled over all consecutive tap positions in the rhythmic patterns, which revealed no significant main effect on synchronization errors. The zero lines represent the onset of stimuli; the zero level means perfect synchronization; and the area below this level corresponds to responses in advance of stimulus presentation. (Tick marks on the abscissas are not a scale; points are connected with lines to aid the eye.) 


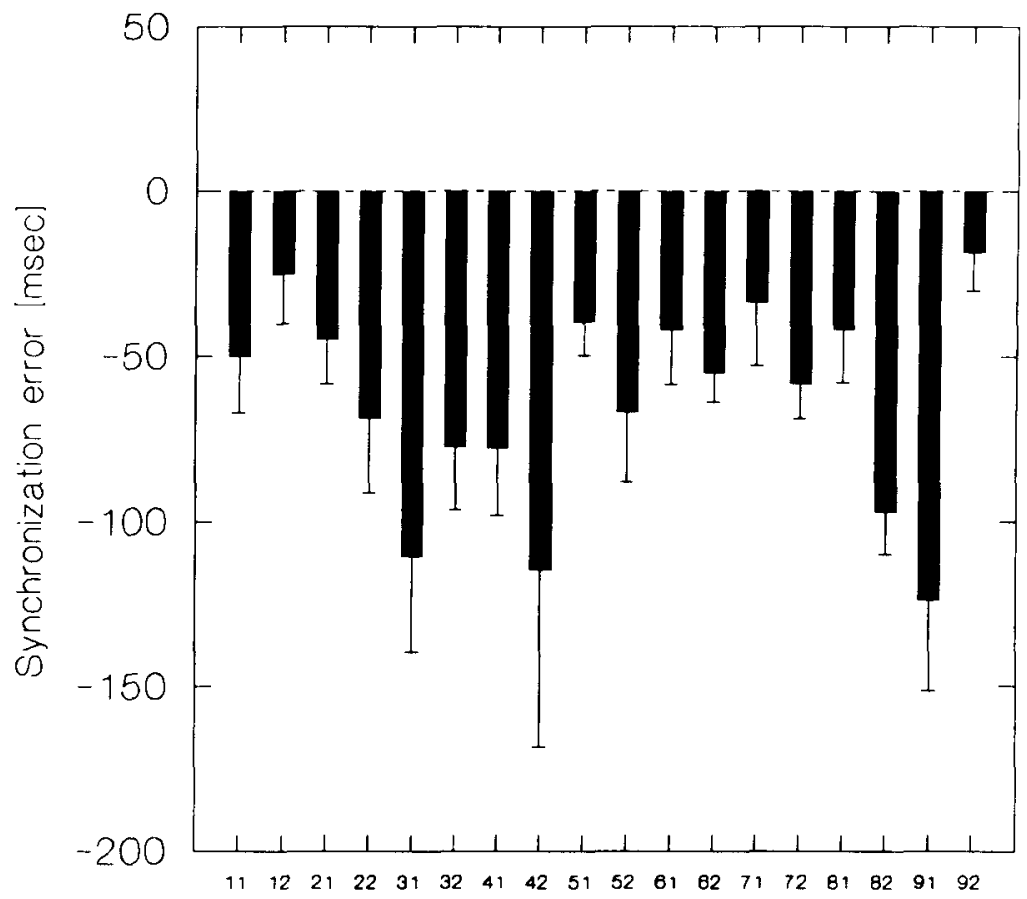

Subject

Figure 8. Individual total means with between-trial-blocks standard deviations of synchronization errors. Subjects' trial means were pooled over all consecutive tap positions in the rhythmic patterns, for both stimulus sequences, and for the four feedback conditions. Abscissa: 11-91, Pairs 1-9, Subject 1; 12-92, Pairs 1-9, Subject 2. The dashed line represents the onset of stimuli; the zero level means perfect synchronization; and the area below this level corresponds to responses in advance of stimulus presentation.

\section{Synchronization Errors}

Linked (cooperative) performances. The systematic advance of responses with respect to stimulus occurrence has been observed in previous synchronization studies (see, e.g., Fraisse, 1978, 1982; Hary \& Moore, 1985; Kolers \& Brewster, 1985; Radil et al., 1990b). Responses to predictable stimuli should be considered as anticipatory not only in the case when they appear objectively in advance with respect to stimuli, but also when the responses are delayed by an interval shorter than the usual reaction time of at least $150 \mathrm{msec}$ (Adams \& Creamer, 1962; Najenson et al., 1989; Schmidt, 1968; Schmidt \& Christina, 1969).

The cause of motor anticipation of a sequential predictable series of stimuli is still obscure (Radil et al., 1990b) and deserves special analysis. Other studies have demonstrated that anticipation is suppressed by shortening the available interstimulus intervals, either through increasing the stimulation rate (Müller, Ilmberger, Pöppel, Mates, \& Radil, 1990) or through increasing the stimulus duration during these intervals (Franěk et al., 1989). A transition from anticipative toward pure reactive (delayed) responses has been observed when the interstimulus interval duration is lengthened above an individ- ual threshold of a few seconds (Najenson et al., 1989; Pöppel, Müller, \& Mates, 1990). On the other hand, anticipation remains present even when some of the tones in the patterns are missing. Moreover, a general tendency to lengthen anticipation under these conditions has been observed (Radil, Mates, Ilmberger, \& Pöppel, 1990a). We also observed that anticipation intervals were significantly longer when sequences were accentuated by frequency rather than by duration changes, and significantly longer under Conditions AFB and CFB than under Conditions NFB and OFB.

The findings above concerning anticipation intervals indicate that greater task "difficulty" (i.e., higher information load as expressed in the absence of some stimuli, or in different frequencies of some tones, or in unpredictability caused by alien feedback) might result in a higher activation, with a likely alteration of corresponding motor programs and/or further decision making, hence requiring additional processing time. Consequently, the duration of anticipation increases.

We do not possess explicit knowledge about the temporal scheme of events taking place under the AFB and CFB conditions. Due to interindividual differences and uncorrelated variance in performance and brain timing in 
both subjects, there might be in both cases a certain temporal incongruity between auditory and tactile-kinaesthetic feedback which makes the programming of rhythmic motor activity more difficult. An additional factor under the CFB condition could be the mutual dynamic influence between both incongruent systems, which further increases the complexity of the programming task for the brain and which is reflected in larger anticipation intervals.

There were no differences in time trends of individual series of synchronization errors, no correlations between these trends, and no cross-correlations between the synchronization error series of "cooperating" subjects in a pair under the experimental conditions studied. There seems to have been no "cooperation" between subjects in the sense of instantaneous unidirectional or bidirectional influence on each other's performance. The influence from the other subject resulted only in an increase of mean anticipation interval without any relation to the instantaneous value of the anticipation interval of that subject. This influence seems to have been established at the beginning of each trial, and it did not change over the sequence.

Separated performances. The objectively measured dissynchrony of motor response and stimulus does not necessarily mean that these events are also dissynchronous in the brain. Let us assume, as Hary and Moore (1985) did, that "the neural events corresponding to the two external events, observed to be dis-synchronous in the laboratory, could occur synchronously at some point in the nervous system"' (p. 78). This synchronous occurrence may also be understood in terms of a "temporal window" of nonzero duration, which can perhaps be created by a neuronal oscillator. One period of such an oscillation may define a time zone (temporal window), within which the before-after relationship is not defined (Pöppel, 1970; Pöppel, Schill, \& von Steinbüchel, 1990). The analogous hypothesis of a "psychological moment"' (Fraisse, 1978) could be considered, too.

Mutual timing in "temporal central availability" (Pöppel, Schill, \& von Steinbüchel, 1990) of the feedback signals and proper auditory stimuli might just be the important factor that determines anticipation, although its exact mechanisms are not understood yet. It is of potential importance that the time of transduction ("conduction delay" in Hary \& Moore, 1985; "perception latency" in Fraisse, 1978) to the cortex for the auditory pathway is shorter than that for the tactile-kinesthetic one, resulting in different temporal central availability of both signals. Under the NFB condition, the longer delay is in the feedback (tactile-kinesthetic) pathway, so that the response observed from the outside has to be in advance of the related stimulus in order that delayed feedback information about it may appear simultaneously (in some of the meanings of simultaneity given above) with the stimulus in the central nervous system.

The faster auditory feedback pathway involved in the OFB condition may have supplied information about one's own response earlier than in the NFB condition, when the subject's brain did rely upon tactile-kinesthetic information only. Therefore, in the former case, the motor command could have been sent later, causing the anticipation intervals to be shorter. Fraisse (1982) mentioned a greater anticipation in synchronization performed by foot than by finger; this can also be explained by the longer time of transduction of peripheral information. Comparison of Conditions NFB (i.e., no additional feedback) and OFB (i.e., own feedback) showed that the auditory feedback derived from the subject's own taps could influence positively his or her performance in the sense that the synchronization errors became smaller; however, significance was not achieved.

Kolers and Brewster (1985) have hypothesized that the use of the tactile feedback information is inconsistent, if there is any at all. Nevertheless, the tactile-kinesthetic feedback coming from the motor effector and from touching the response key remained constant across experimental conditions; only the external auditory feedback loop created additional variance. Thus, the tactile-kinesthetic feedback could have determined the results in all the experimental conditions and could have suppressed the expected positive influence of additional auditory feedback. It resulted in a nonzero anticipation even in the OFB condition, where both signals were coming through the same sensory pathway (i.e., with the same delay). Persisting anticipation when both the feedback signal and the stimulus were in the visual modality has also been observed by Kolers and Brewster. They found a larger variability of synchronization errors in the intramodal (visual) stimulus and feedback setting as opposed to the intermodal (visual-tactile) condition; the mean values were not different. It might be possible that subjects use the available feedback information according to the accuracy that it offers for their judgment about the stimulus-response synchrony. The larger variability mentioned can be a result of a more frequent intervention of feedback regarding the subject's strategy in the intramodal condition, in accordance with this "accuracy"-determined preference. Nevertheless, it is obvious that feedback can directly influence the timing of subsequent movements only and not those that have already been executed. Rather, the instantaneous feedback information in performing a sequence of repetitive motor acts is used to tune or update programs of corresponding motor- and timing-control systems.

We found that in Conditions NFB and OFB the average anticipation interval was somewhat longer than what we observed in the previous study, in which the tapping of single subjects was investigated and no additional signals were provided (Radil et al., 1990b). This was probably caused by the fact that synchronization errors vary interindividually, as can be seen from the individual results shown in Figure 7.

\section{Intertap Intervals}

The individual intertap intervals shown in Figure 3 support the hypothesis that different strategies used for timing of rhythmic patterns result in subjective deformation 
of regular patterns (Franěk et al., 1991b). Franĕk and coworkers discussed the systematic shortening of the second intertap interval in comparison with the first one in the patterns as one possible strategy used in rhythmic patterns. Both the results of experiments performed by Franèk et al. (1991b), in which various frequency accents were used, and the present observed differences in the timing of intertap intervals in the patterns between the $D$ and $F$ sequences show that these strategies are influenced by the type of accent. In $63 \%$ of the cases, our subjects used the long-short strategy (i.e., they made the first intertap interval longer than the second one) for the $\mathrm{D}$ sequence. The prevailing use of this strategy might be connected with the differences in tap durations in the patterns observed for this sequence (see below).

In the study by Franěk et al. (199lb) a lengthening of the fifth intertap interval in six-tone patterns was also observed. It was suggested that the prolongation was caused by a subjective accentuation that was needed as a control point for timing at the end of a temporal interval, reflecting the duration of a "psychological present" (Fraisse, 1984 ) or the limited capacity of a "central integration mechanism"' (e.g., Pöppel, 1988). Such a subjective accentuation might be one of the sources of the (nonsignificant) prolongation of the fourth intertap interval that was observed in our experiment for the D sequence as well.

The present observed distortions in intertap interval durations were not influenced by the type of auditory feedback condition used.

\section{Tap Durations}

A brief discussion of further distortions in performance timing that were not influenced by the type of external feedback adopted is in order. The subjects were always instructed to tap synchronously only with stimulus onsets and not to follow the duration of stimulus tones. Despite this instruction, we observed a systematic decrease of tap durations in the reproductions of the $\mathrm{D}$ sequence, where the first tap, corresponding to the accentuated tone, was the (significantly) longest tap in the patterns. No such phenomenon was observed for the $\mathrm{F}$ sequence, where the first tone was accentuated by frequency changes. However, in both sequence types, the fourth tap was the (significantly) shortest one. The technique that we used did not allow us to measure the tapping force; stronger taps could be assessed in terms of the lengthening of the tap durations only. The results could mean that the differences in duration of the differing tones are perceived as stronger accents than are the differences in frequency. On the basis of the observed (nonsignificant) prolongation of the last (i.e., the fourth) intertap interval in the patterns for the $D$ sequence, it could be assumed that in connection with the perceived stronger accent, there is also a stronger tendency to group tones in the rhythmic patterns together. This grouping tendency is reflected by the separation of patterns by longer pauses. The shortening of the second intertap interval for the D sequence might be a subjective compensation for the longer first tap duration in the patterns, although the durations of the first intertap intervals did not differ from the interstimulus interval for this sequence. The striking interindividual differences in tap durations evident in Figure 5 might reflect different levels in the subjects' musical skill. It has already been reported (Franèk et al., 1991a) that musical skill influences tap durations.

\section{REFERENCES}

Adams, J. A., \& Crenmer, L. R. (1962). Proprioception variables as determiners of anticipatory timing behavior. Human Factors, 4, 217-222.

Clynes, C. M., \& WAlker, J. (1982). Neurobiologic functions of rhythm, time and pulse in music. In M. Clynes (Ed.), Music, mind and brain: The neuropsychology of music (pp. 171-216). New York: Plenum.

Fraisse, P. (1978). Time and rhythm perception. In E. C. Carterette \& M. Friedman (Eds.), Handbook of perception: Vol. 8. Perceptual coding (pp. 203-254). New York: Academic Press.

Fraisse, P. (1982). Rhythm and tempo. In D. Deutsch (Ed.), Psychology of music (pp. 149-180). New York: Academic Press.

Fraisse, P. (1984). Perception and estimation of time. Annual Review of Psychology, 35, 1-36.

Franě,, M., Mates, J., Radil, T., Beck, K., \& Pöppel, E. (1991a). Finger tapping in musicians and nonmusicians. International Journal of Psychophysiology, 11, 277-279.

Franě, M., Mates, J., Radil, T., Beck, K., a Pöppel, E. (1991b). Sensorimotor synchronization: Motor responses to regular auditory patterns. Perception \& Psychophysics, 49, 509-516.

Franek, M., Radil, T., Pöppel, E., Beck, K., Mates, J., IlmBERGER, J. (1989). Following of rhythmic tonal sequences by tapping and holding the response key. Activitas Nervosa Superior, 31, $152-153$.

Gallo, P. S., JR., \& McCuntock, C. G. (1965). Cooperative and competitive behavior in mixed-motive games. Journal of Conflict Resolution, 9, 68-78.

HARY, D., \& MOORE, G. P. (1985). Temporal tracking and synchronization strategies. Human Neurobiology, 4, 73-77.

Ilmberger, J., Müller, U., Pöppel, E., Mates, J., \& Radil, T. (1990). Timing of rhythmic tapping and lateralization. Activitas Nervosa Superior, 32, 142-143.

KNIGHT, J. L., JR. (1987). Manual control and tracking. In G. Salvendy (Ed.), Handbook of human factors (pp. 182-218). New York: Wiley.

Kolers, P. A., Brewster, J. M. (1985). Rhythms and responses. Journal of Experimental Psychology: Human Perception \& Performance, 11, 150-167.

MARWELL, G., \& SCHMITT, D. R. (1975). Cooperation: An experimental analysis. New York: Academic Press.

MATES, J. (1990). A system of personal computer control programs for tapping experiments. Computer Methods \& Programs in Biomedicine, 33, 43-48.

MATES, J. (1991). Extending the model of self-paced periodic responding: Comment on Vos and Ellermann (1989). Joumal of Experimental Psychology: Human Perception \& Performance, 17, 877-879.

Müller, U., Ilmberger, J., Pöppel, E., Mates, J., \& Radil, T. (1990). Stimulus anticipation and the $30 \mathrm{~ms}$ basic timing unit during rhythmic tapping. Activitas Nervosa Superior, 32, 144-145.

Najenson, T., RoN, S., \& Behroozi, K. (1989). Temporal characteristics of tapping responses in healthy subjects and in patients who sustained cerebrovascular accident. Brain, Behavior \& Evolution, 33, 175-178.

Peters, M. (1989). The relationship between variability of intertap in tervals and interval duration. Psychological Research, 51, 38-42.

PöPPEL, E. (1970). Excitability cycles in central intermittency. Psychologische Forschung, 34, 1-9.

Pöppel, E. (1988). Mindworks: Time and conscious experience. Boston: Harcourt Brace Jovanovich.

Pöppel, E., Müller, U., \& Mates, J. (1990, October). Temporal constraints in synchronization of motor responses to a regular sequence 
of stimuli. Paper presented at the meeting of the Society for Neuroscience, St. Louis.

Pöppel, E., Schill, K., \& von Steinbuchel, N. (1990). Sensory integration within temporally neutral systems states: A hypothesis. Naturwissenschaften, 77, 89-91

Radil, T., Mates, J., Ilmberger, J., \& Pöppel, E. (1990a). Interpolation and extrapolation during rhythmical tapping. Activitas Nervosa Superior, 32, 75-76.

Radil, T., Mates, J., Ilmberger, J., \& Pöppel, E. (1990b). Stimulus anticipation in following rhythmic acoustical patterns by tapping. Experientia, 46, 762-763.

Rapoport, A., Chammak, A. M. (1965). Prisoner's dilemma: A study in conflict and cooperation. Ann Arbor: University of Michigan Press.

Rosenberg, S., \& Hall, R. L. (1958). The effect of different social feedback conditions upon performance in dyadic teams. Joumal of Abnormal \& Social Psychology, 57, 271-277.

SAUTER, S., \& SMITH, K. U. (1971). Social feedback: Quantitative division of labor in social interactions. Journal of Cybernetics, 1, 80-93.

ShafFer, L. H. (1984). Timing in solo and duet piano performances. Quarterly Journal of Experimental Psychology, 36A, 577-595.

SCHMIDT, R. A. (1968). Anticipation and timing in human motor performance. Psychological Bulletin, 70, 631-646.

Schmidt, R. A., \& Christina, R. W. (1969). Proprioception as a mediator in the timing of motor responses. Journal of Experimental Psychology, 81, 303-307.

SMEAD, A. (1972). Cooperation and competition. In L. S. Wrightsman (Ed.), Social psychology in the seventies (pp. 131-155). Belmont: Brooks/Cole.

SMELSER, W. T. (1961). Dominance as a factor in achievement and perception in cooperative problem solving interactions. Journal of $A b$ normal \& Social Psychology, 62, 535-542.

SMIth, K. U., \& ARNDT, R. (1969). Experimental hybrid-computer automation in perceptual-motor and social behavioral research. Journal of Motor Behavior, 1, 11-28.
Smith, K. U., KAO, H. (1971). Social feedback: Determination of social learning. Journal of Nervous \& Mental Disease, 152, 289-297.

SмIтн, T. J., \& SMIтH, K. U. (1987a). Feedback-control mechanisms of human behavior. In G. Salvendy (Ed.), Handbook of human factors (pp. 251-293). New York: Wiley.

Sмith, T. J., SMITH, K. U. (1987b). Motor feedback control of human cognition-implications for the cognitive inference. In G. Salvendy, S. L. Sauter, J. J. Hurrel, Jr. (Eds.), Social, ergonomic and stress aspects of work with computers (pp. 239-254). Amsterdam: Elsevier.

VorberG, D., Н НамвUCh, R. (1984). Timing of two-handed rhythmic performance. In J. Gibbon \& L. Allan (Eds.), Timing and time perception (Annals of The New York Academy of Sciences, Vol. 423, pp. 390-406). New York: New York Academy of Sciences.

Vos, P. G. Ellermann, H. H. (1989). Precision and accuracy in the reproduction of simple tone sequences. Joumal of Experimental Psychology: Human Perception \& Performance, 15, 179-187.

WAGNER, N., ZEAMAN, D. (1956). Team and individual performances on a motor learning task. Joumal of General Psychology, 55, 127-142.

WichmaN, H. (1970). Effects of isolation and communication on cooperation in a two-person game. Journal of Personality \& Social Psychology, 16, 114-120.

Wing, A. M., KeEle, S. MARgolin, D. I. (1984). Motor disorder and the timing of repetitive movements. In J. Gibbon \& L. Allan (Eds.), Timing and time perception (Annals of The New York Academy of Sciences, Vol. 423, pp. 183-197). New York: New York Academy of Sciences.

Wing, A. M., \& Kristofferson, A. B. (1973). Response delays and the timing of discrete motor responses. Perception \& Psychophysics, 14, 5-12.

(Manuscript received March 6, 1991; revision accepted for publication May 26, 1992.) 